\title{
Rare case fulminant hepatic failure
}

\section{Introduction}

Fulminant hepatic failure is acute liver failure complicated by encephalopathy that occurs within 8 weeks after the onset of symptoms. The most common causes in the U.S.A are viral infections and hepatotoxic drugs. Neoplasms have been recognized as a potential cause of this syndrome, but is often unrecognized in the absence of known history of malignancy. The case describes a patient with multi factorial etiologies of liver failure and with characteristic findings on imaging and pathology that highlights an approach to patients with this syndrome.

\section{Case report}

53 year old Caucasian male presented with non-productive cough and orthopnea since 7 days, abdominal discomfort - localized to right lower quadrant, followed by abdominal distention and yellowing of eyes and urine since 4 days. Consumed 50 tablets of Tylenol to relieve abdominal pain. His past medical history was relevant for end stage COPD, he was a current smoker with 15 pack year smoking history, and consumed $10-15$ beers per week. Family history relevant for liver disease of unknown etiology in his mother. Social history 15 pack year smoking history drinks $10-15$ beers per week. Physical examination relevant for evidence of facial wasting, citric sclera, distended abdomen with fullness in RUQ. Admission labs significant for mild uremia BUN/Cr 47/0.5, bicarbonate 22, anion gap 17, AST/ ALT 689/403, amylase 32, alkaline phosphatase 1018, lipase 218, ammonia 113 , ethanol $<10$, bilirubin 5.8 , Salicylate $<1$, lactate 4.3 , Tylenol $<10$, ABG -7.36/42/83/23.6/96\% 21 nasal cannula. Chest $x$-ray showed evidence of increased fullness of left hilum and $\mathrm{Ct}$ abdomen showed heterogeneously enhancing nodular enlarged liver with small as cites and enlarged lymph nodes in Porto hepatic region related to hepatic cirrhosis vs a diffuse infiltrating neoplastic process. ${ }^{1}$

\section{Hospital course}

Patient admitted to ICU and started on mucomyst infusion for presumed liver failure from Tylenol toxicity with possibility of underlying alcoholic liver disease. Transplant center contacted for early referral and possible transplant. ${ }^{2}$ Patient was not accepted, per recommendation started on lactulose, albuterol, pulmicort, Cefotaxime for spontaneous bacterial peritonitis prophylaxis.

On day 2, he was complaining of increasing shortness of breath and abdominal discomfort. Labs showed AST/ALT 699/420, ammonia-69, bilirubin -7.7, inr1.45, lactate-6.1. MRI abdomen doneinnumerable hepatic masses suspicious for hepatic metastasis or multifocal hepatocellular carcinoma with hepatic cirrhosis. Patient was planned for CT guided liver biopsy. Vitamin K and FFP were administered overnight.

On day 3 patient developed worsening hyponatremia $\mathrm{Na} 128$ and transaminitis AST/ALT 1381/706, alkaline phosphatase 980, inr1.86, ammonia 113, bilirubin 8.2 , lactate 6.4 .

On day 4 patient increasingly dyspneic and tachypnieic with worsening hypoxemia. He was taken to Intervention Radiology suite
Volume 2 Issue 6 - 2017

\author{
Nisha Kotecha \\ Division of Pulmonary, Critical Care and Sleep Medicine, Atlantic \\ Health System, USA
}

Correspondence: Nisha Kotecha, Division of Pulmonary, Critical Care and Sleep Medicine, Atlantic Health System, USA, Email nisha.kotecha@atlantichealth.org

Received: April 13, 2017 | Published: May 18, 2017

for CT guided biopsy and subsequently developed acute respiratory distress needing intubation. He developed circulatory shock and was started on vasopressors, levophed and dopamine. He developed oliguria and hyperthermia, he was started on empiric broad spectrum antibiotics- cefepime, vancomycin and levaquin for concern for sepsis. He received calcium gluconate and insulin to correct hyperkalemia. Urgent CVVH was planned. Hemodynamically unstable on maximum dose of levophed and dopamine. Labs with worsening renal function, and transaminitis, BUN/Cr 76/2.5, AST/ALT 24367/3876, Persistent lactic acidosis-7.8. Patient goes into ventricular tachycardia with pulseless arrest. ACLS protocol initiated. Unable to resuscitate patient after 30 minutes.

Autopsy requested by wife. Pathology results; Liver biopsy metastatic small cell carcinoma with metastasis to peribronchial lymph nodes. Subtotal replacement of liver, spleen and bone marrow, acute tubular necrosis of kidney, severe emphysema with bilateral bullae formation, normal brain.

\section{Discussion}

Fulminant hepatic failure is defined as acute liver failure complicated by encephalopathy that occurs within 8 weeks after the onset of symptoms. The most common causes in the U.S.A are viral infections and hepatotoxic drugs. Neoplasms have been recognized as a potential cause of this syndrome.

Malignant diseases involving the liver and manifesting as acute liver failure include breast carcinoma, leukemia, malignant melanoma, small cell lung cancer (SCLC), gastric carcinoma, prostate carcinoma, pancreatic carcinoma, lymphoma, and hemangioendothelioma and Non Hodgkins lymphoma. ${ }^{3}$

\section{SCLC}

SCLC Comprise about $25 \%$ of all lung cancers. They mostly appear as hilar masses on chest x-ray. Approximately $70 \%$ initially present with extensive stage disease. It has a propensity to spread to liver (most common), adrenals, bone, bone marrow and brain.

\section{Liver metastasis}

Rapid progression to fulminant hepatic failure is rarely the result of diffuse liver infiltration. High mortality due to early development 
of multiple organ failure symptoms. Cause-obstruction of hepatic vessels by tumor deposits leads to edema and ischemic necrosis of centrally located hepatocytes. Metastatic embolization is also a possible hypotheses.

\section{Symptoms}

Patients commonly present with Malaise 50\%, Weight loss 39\%, Ruq pain 39\%, Fever 33\%, Hyperbilirubinemia, Mild transaminitis, Elevated LDH and Hyperuricemia.

\section{Approach}

Liver parenchyma abnormalities necessitates a thorough evaluation to exclude neoplastic disease.

Physical should include examination of skin for melanoma, breast examination, consider cheat x-ray or CT chest in persons with risk factors for lung cancer. Endoscopy and abdominal CT scan may aid in diagnosis of intra-abdominal neoplasm. Once FHF develops due to diffuse liver metastasis prognosis is dismal with the syndrome ultimately resulting in death in 6 to ten days. Death is invariably caused by multiorgan system failure. Clinical evidence of sepsis or radiological evidence of pneumonia are present 24 hours prior to death. Presence of malignancy is an absolute contraindication to transplant.

\section{Conclusion}

Diffuse liver metastasis must be considered when imaging modalities show hepatomegaly in patients with fulminant hepatic failure especially when viral and toxic hepatitis are ruled out. Our case was particularly challenging given the presence of a known hepatotoxin acetaminophen. The pointers in the case were the characteristic chest $\mathrm{x}$-ray, smoking history and imaging of the liver.

\section{Radiology}

\section{Chest X-ray}

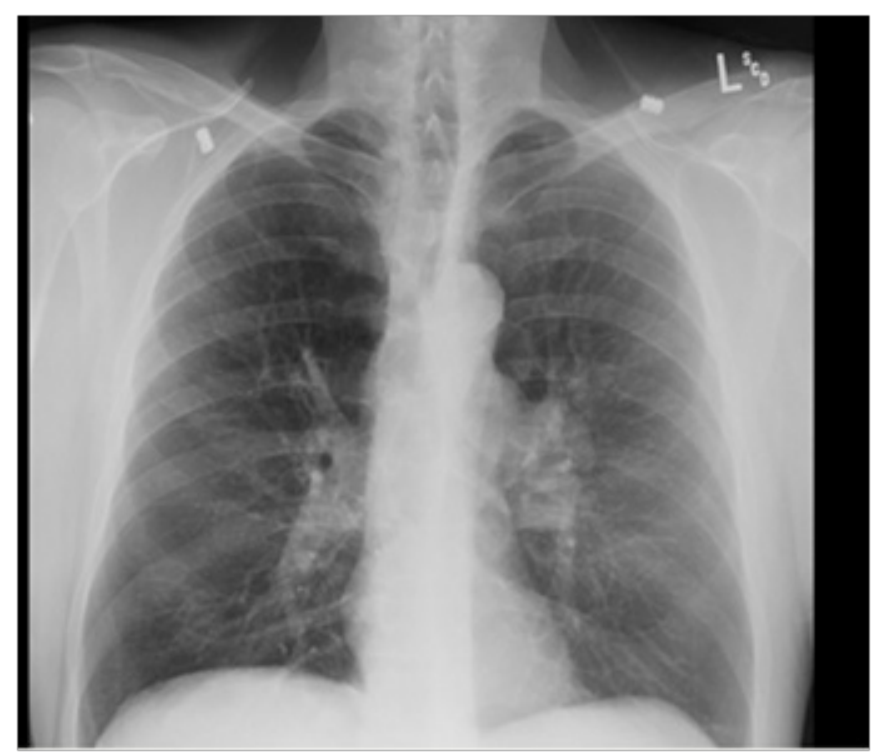

Figure I Chest X-ray.

\section{CT abdomen}

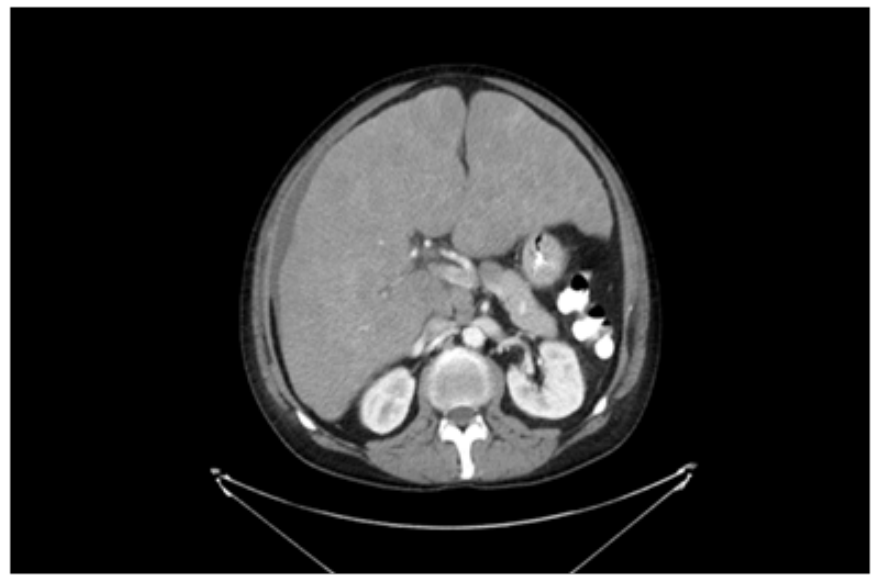

Figure $2 \mathrm{CT}$ abdomen.

\section{MRI abdomen}

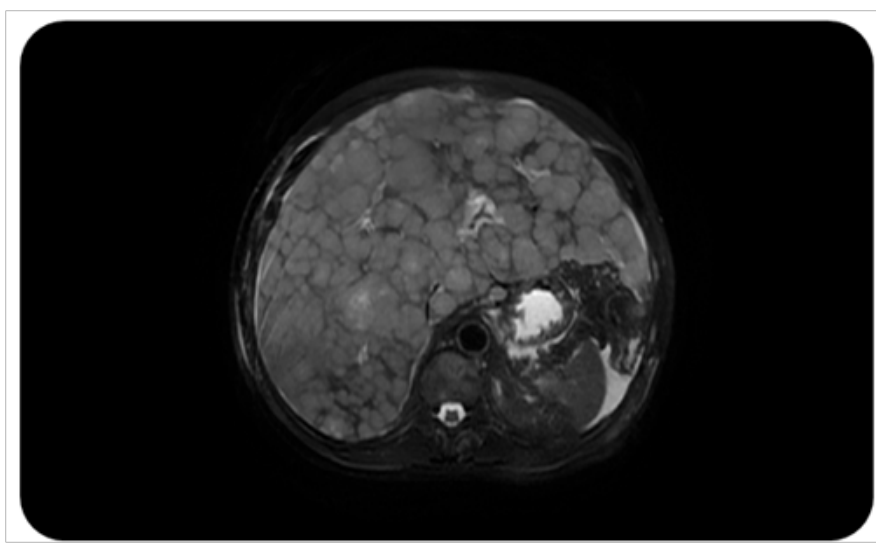

Figure $3 \mathrm{MRI}$ abdomen

\section{Acknowledgements}

None.

\section{Conflict of interest}

The author declares no conflict of interest.

\section{References}

1. D Rowbotham, J Wendon, R Williams. Acute liver failure secondary to hepatic infiltration: a single centre experience of 18 cases. Gut. 1998;42(4):576-580.

2. William Lee. Etiologies of acute liver failure. Semin Liver Dis. 2008;28(2):142-152.

3. Ostapowicz G, Fontana RJ, Schiødt FV, et al. Results of a prospective study of acute liver failure at 17 tertiary care centers in the United States. Ann Intern Med. 2002;137(12):947-954. 